\title{
Dynamic evolution of Panax species
}

\author{
Hyeonah Shim ${ }^{1} \cdot$ Nomar Espinosa Waminal $^{2} \cdot$ Hyun Hee Kim ${ }^{2} \cdot$ Tae-Jin Yang $^{1}$ (])
}

Received: 5 January 2021 / Accepted: 8 January 2021 / Published online: 20 February 2021

(c) The Author(s) 2021

\begin{abstract}
Background Panax ginseng is one of the most valuable medicinal plants in Korea. However, deciphering its full genome sequence information for crop improvement has been hampered due to its complex genomic, genetic, and growth characteristics. Many efforts have been made in the past decade to overcome these limitations and understand the genome structure and the evolutionary history of $P$. ginseng.

Methods This review aims to discuss the current status of genomic studies on P. ginseng and related species, and the experimental clues suggesting phylogenetic classification and evolutionary history of the genus Panax.

Conclusion The development of sequencing technologies made genome sequencing of the large $P$. ginseng genome possible, providing fundamental information to deciphering the evolutionary history of $P$. ginseng and related species. $P$. ginseng went through two rounds of whole genome duplication events after diverging from the closest family Apiaceae, which was unveiled from complete whole genome sequences. Further in-depth comparative genome analysis with other related species and genera will uncover the evolutionary history as well as important morphological and ecological characteristics of Panax species.
\end{abstract}

Keywords Panax ginseng $\cdot$ Evolution $\cdot$ Whole genome sequence $\cdot$ Whole genome duplication

\section{Introduction}

Panax ginseng (named Korean ginseng or Asian ginseng) is one of the most valuable representative medicinal plants in Korea, which is also known worldwide. Ginseng, along with other Panax species distributed in Asia and North America, belongs to the genus Panax of the Araliaceae family, which contains around 1,500 species (Plunkett et al. 1996; Wen et al. 2001). Ginseng also naturally grows in parts of China and Russia (Grushwitsky 1961; Wen and Zimmer 1996; Zhuravlev et al. 2008). It has $2 n=4 x=48$ chromosomes (Waminal et al. 2012) with an allotetraploid genome of $\sim 3.6 \mathrm{Gbp}$ (Kim et al. 2018). Ginseng genetic studies, and breeding in general, are quite challenging due to its limited

Tae-Jin Yang

tjyang@snu.ac.kr

1 Department of Agriculture, Forestry and Bioresources, Plant Genomics and Breeding Institute, Research Institute of Agriculture and Life Sciences, College of Agriculture and Life Sciences, Seoul National University, 1 Gwanak-ro, Gwanak-gu, Seoul 08826, Korea

2 Department of Chemistry and Life Science, Bioscience Institute, Sahmyook University, Seoul 01795, Republic of Korea growth characteristics such as a long generation time of four years and only a few seeds $(\sim 40)$ set per plant starting from the fourth year (Choi et al. 1998; Choi 2008; Jayakodi et al. 2014).

The Panax genus comprises about 17 species (Zhang et al. 2020). Panax quinquefolius L., also known as North American ginseng (Kim et al. 2016b), grows in North America along with Panax trifolius (Wen and Zimmer 1996). Panax notoginseng Burkill grows and is cultivated in China (Schorger 1969; Wen and Zimmer 1996), while Panax vietnamensis Ha et Grushv exists in certain regions of Vietnam (Van Duy et al. 2016). These Panax species are cultivated in their respective countries for their high medicinal value and scarcity.

Here, we review the genomics research in ginseng and the related species, with special emphasis on the whole genome assembly status, genomic characteristics in Panax species as well as the evolution of Panax species as revealed by comparative genomics analyses. We also present the on-going genomics studies in related species outside the genus Panax but within the Araliaceae family. 


\section{Status of genomic research in the genus Panax}

Currently, whole genome sequence information is available only for two species, the allotetraploid $P$. ginseng (Kim et al. 2018) and the diploid P. notoginseng (Chen et al. 2017; Zhang et al. 2017). Xu et al. (2017) assembled the whole $P$. ginseng genome and obtained a $3.41 \mathrm{Gbp}$ draft assembly with a scaffold N50 of $108.71 \mathrm{~kb}$ and annotated 42,006 genes. In 2018, a draft sequence of the major $P$. ginseng cultivar of Korea, 'Chunpoong', was completed and published (Kim et al. 2018). This draft sequence consisted of $2.98 \mathrm{Gbp}$ with a scaffold N50 of $569.02 \mathrm{~kb}$ and 59,352 annotated genes. Sequence and annotation information as well as basic genomic analysis tools provide a golden standard via an open-access platform database for researchers around the world to utilize for ginseng research (Jayakodi et al. 2018).

Other genomic studies on the whole genome sequence are available for $P$. notoginseng. In 2017, Zhang et al. completed a $1.85 \mathrm{~Gb}$ whole genome with a scaffold N50 of $157.81 \mathrm{~kb}$ and annotated 34,369 genes (Zhang et al. 2017). In the same year, Chen et al. reported a $2.39 \mathrm{~Gb}$ whole genome sequence with a scaffold N50 of $96 \mathrm{~kb}$, annotating 36,790 genes (Chen et al. 2017). In 2020, Fan et al. completed a $2.25 \mathrm{~Gb}$ assembly consisting of 16,469 scaffolds with a contig N50 $220.89 \mathrm{~kb}$ and annotated 39,452 genes (Fan et al. 2020). Jiang et al. reported a significantly improved chromosome-level assembly of $P$. notoginseng. The $2.66 \mathrm{~Gb}$ reference genome has a scaffold N50 of $216.47 \mathrm{Mb}$ with 37,606 genes annotated (Jiang et al. 2020). The assembly statistics of the $P$. ginseng and $P$. notoginseng genome are provided in Table 1.

\section{Molecular cytogenetic tools in ginseng genome analysis}

Genomic features can be analyzed through molecular cytogenetics in which genome size, chromosome number, and clues to understand genome evolution can be obtained.
With the development of cytogenetic tools such as fluorescence in situ hybridization (FISH) (Pinkel et al. 1986), it became much easier to analyze the karyotype of plant species and utilize them for taxonomic studies. The first $P$. ginseng karyotype was reported by Waminal et al. (2012), which was later improved by the identification of abundant satellite DNA, Pg167TR (Choi et al. 2014; Waminal et al. 2017). Identification of additional repeat elements widened the scope of cytogenetic markers for ginseng genome analysis (Fig. 1).

Advances in cytogenetics also made possible the sequencing and analysis of individual chromosome through flow sorting of chromosomes (Doležel et al. 2014). Some example applications of sequencing of flow-sorted chromosomes include cereal crops such as Triticum aestivum (IWGSC 2014), T. dicoccoides (Akpinar et al. 2018), and Hordeum vulgare L. (Lysák et al. 1999). Coupling flow sorting with sequencing techniques provides a great platform for highquality genome sequencing and analysis for plants, particularly those with large genomes because many plant species usually have complex genome structures, large genome sizes, and high repetitive content as well as high levels of heterozygosity.

\section{Two whole genome duplication events in Panax ginseng}

Structure and characteristics of ginseng genome were elucidated by the draft genome sequence of $P$. ginseng (Kim et al. 2018). Although the available genome assemblies are not in chromosome or pseudochromosome levels, the scaffold information holds critical information and clues on duplication events throughout the evolutionary history of $P$. ginseng. Whole genome assembly resulted in highly homologous sequences between scaffolds and paralogous genes with similarities up to $99 \%$. Reference-guided superscaffolding, using the Daucus carota genome $(2 n=2 x=18)$ as a reference, indirectly suggested that $P$. ginseng went through two rounds of genome duplications independent from those that occurred in the carrot genome. Moreover,

Table 1 Whole genome sequences available for Panax species

\begin{tabular}{|c|c|c|c|c|c|c|}
\hline & \multicolumn{2}{|l|}{ Panax ginseng } & \multicolumn{4}{|l|}{ Panax notoginseng } \\
\hline & Xu et al. (2017) & Kim et al. (2018) & Chen et al. (2017) & Zhang et al. (2017) & Fan et al. (2020) & Jiang et al. (2020) \\
\hline No. of scaffolds & 337,439 & 9845 & 122,131 & 76,517 & 16,469 & 219 \\
\hline Total length & $3.41 \mathrm{~Gb}$ & $2.98 \mathrm{~Gb}$ & $2.39 \mathrm{~Gb}$ & $1.85 \mathrm{~Gb}$ & $2.25 \mathrm{~Gb}$ & $2.66 \mathrm{~Gb}$ \\
\hline Contig N50 & $22.00 \mathrm{~kb}$ & $22.54 \mathrm{~kb}$ & $16 \mathrm{~kb}$ & $13.16 \mathrm{~kb}$ & $220.89 \mathrm{~kb}$ & $1.12 \mathrm{Mb}$ \\
\hline Scaffold N50 & $108.71 \mathrm{~kb}$ & $569.02 \mathrm{~kb}$ & $96 \mathrm{~kb}$ & $157.81 \mathrm{~kb}$ & - & $216.47 \mathrm{Mb}$ \\
\hline Longest scaffold & $1.30 \mathrm{Mb}$ & $3.64 \mathrm{Mb}$ & $834.33 \mathrm{~kb}$ & $1.19 \mathrm{Mb}$ & $7.10 \mathrm{Mb}$ & $295.55 \mathrm{Mb}$ \\
\hline No. of genes & 42,006 & 59,352 & 36,790 & 34,369 & 39,452 & 37,606 \\
\hline
\end{tabular}




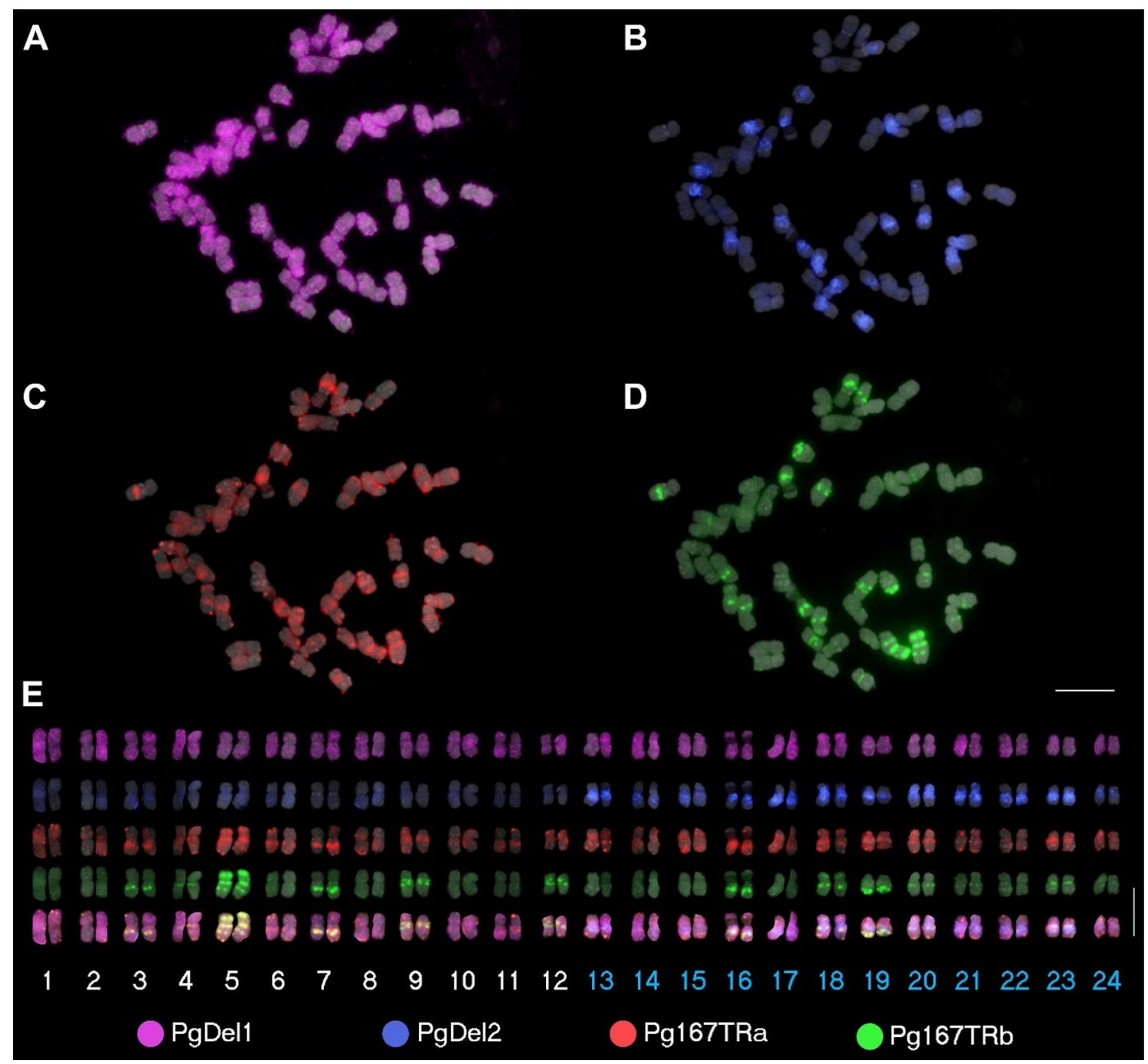

Fig. 1 Simultaneous detection of repeat elements in $P$. ginseng chromosomes using multi-color FISH. FISH signals are shown for transposable element (a, b), and satellite DNA (c, d) probes. a PgDell-Cy5, b PgDel2-DEAC, c Pg167TRa-Texas Red, and

cytogenetic analysis of the genic regions of scaffolds showed that the probe signals appeared in the same chromosome as well as different chromosomes. These genomic characteristics derived from sequence and chromosome analyses of $P$. ginseng suggested a duplication event.

Duplication events were confirmed by synonymous substitution (Ks) values calculated between orthologous gene clusters collected from $P$. ginseng and four dicots that include Arabidopsis thaliana, Vitis vinifera, Solanum lycopersicum, and Daucus carota. Ks values suggested a divergence event between Araliaceae and Apiaceae around 51 million years ago (mya). Moreover, whole genome duplication events of $P$. ginseng-an ancient genome duplication around 28 mya common to all Araliaceae species, and a relatively recent event around 2.2 mya unique d Pg167TRb-Alexa Fluor 488. e Chromosomes from a-d were arranged according to PgDel2 signal and chromosome length. The last row in e show the merged signals of the four repeats. Bars $=10 \mu \mathrm{m}$ (color figure online)

to Panax species-have been inferred from Ks calculation of paralogous gene pairs in $P$. ginseng (Fig. 2) (Kim et al. 2018). Divergence time estimation results were consistent with previous estimations using expressed sequence tags (Choi et al. 2013), repeat composition (Choi et al. 2014), and complete chloroplast genome and nrDNA sequences (Kim et al. 2017). 


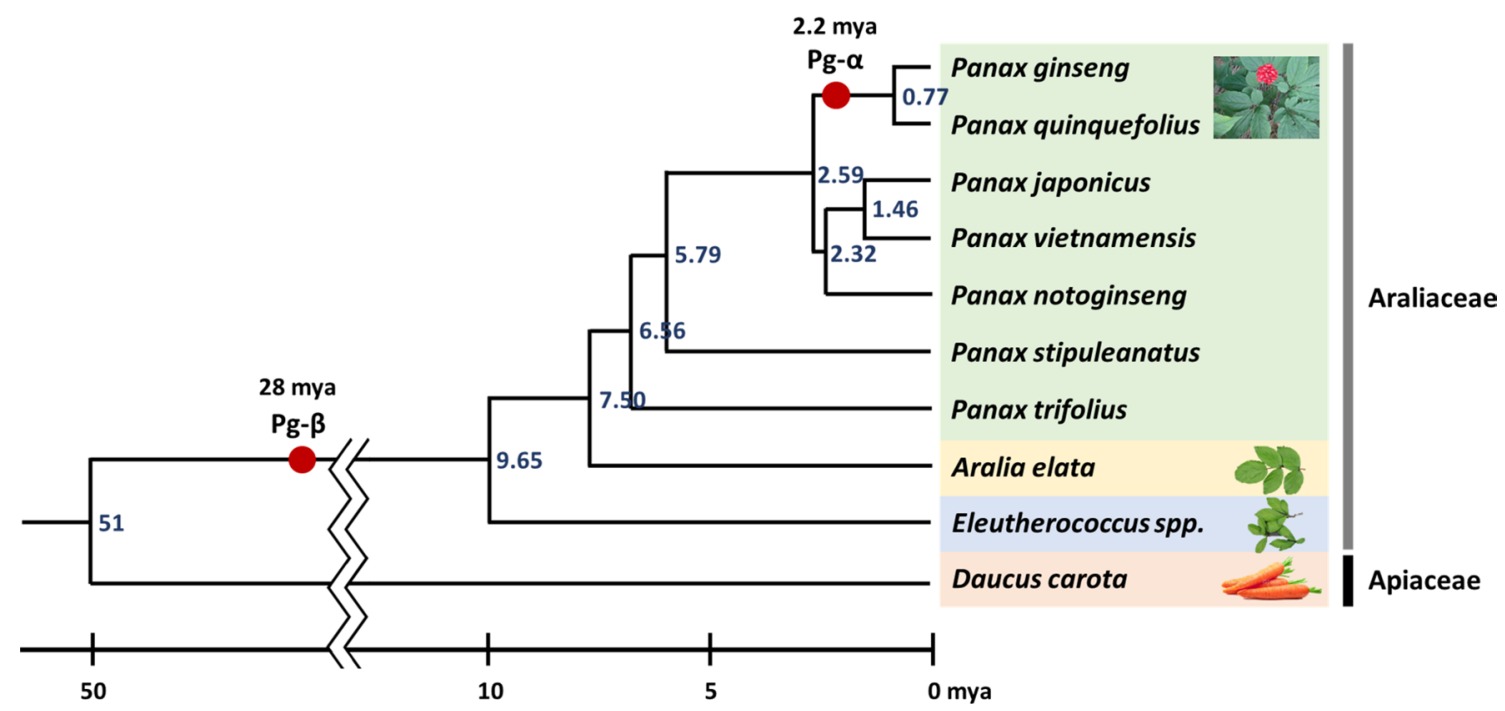

Fig. 2 Two whole genome duplication events occurring for Panax species. Reorganized and redrawn based on divergence time estimations of Lee et al. (2017), Kim et al. (2017), and Kim et al. (2018)

\section{Repeat explosion contributed to the speciation, adaptation, and genome size expansion in Panax}

Major repeat components were first identified by sequencing the bacterial artificial chromosome (BAC) sequences of ginseng (Choi et al. 2014; Jang et al. 2017). Analyses showed that most of the BAC sequences were comprised of repeats, and the two main repeat types were Ty3/Gypsy, consisting of PgDel, PgTat, PgAthila (Choi et al. 2014; Jang et al. 2017), and Ty1/Copia which contains PgTork and PgOryco (Choi et al. 2014). The repeat proportion within the genome could be calculated by mapping whole genome shotgun sequence reads, which showed that $\mathrm{PgDel}$ is the most abundant, $P g$ Dell sub-family being the most abundant (about 25\%) in $P$. ginseng (Lee et al. 2017). The distribution has been visualized by FISH (Fig. 1) (Choi et al. 2014; Lee et al. 2017). Moreover, FISH analysis showed that $P g D e l l$ is distributed across all 24 chromosome pairs while $P g D e l 2$ occupies only half of the chromosome complement; it was detected in only 12 chromosome pairs (Fig. 3) (Choi et al. 2014). This acts as strong evidence of the recent allotetraploidization event for $P$. ginseng emergence (Choi et al. 2014).

The major repeats found in $P$. ginseng were also calculated in other Panax species. Five Panax species and Aralia elata were analyzed for their repeat content, and PgDell showed the most dynamic proportion in the six species analyzed. PgDell was even more abundant in $P$. quinquefolius, covering almost

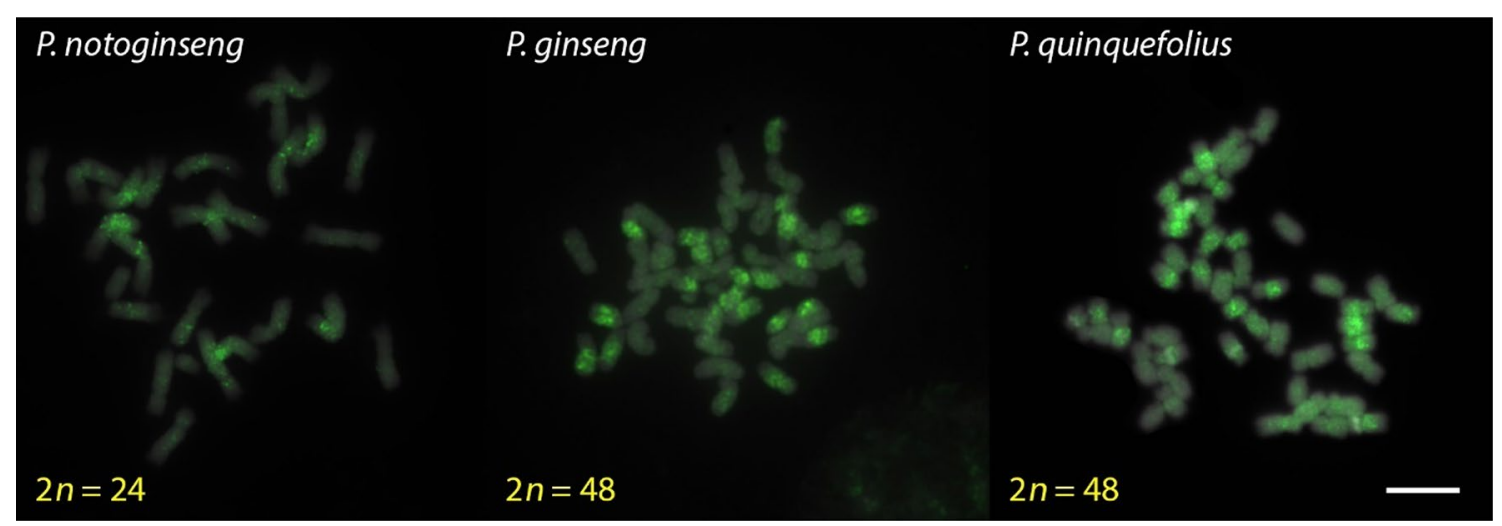

Fig. 3 Distribution of $P g D e l 2$ retrotransposon in diploid P. notoginseng and tetraploid $P$. ginseng and $P$. quinquefolius. In $P$. notoginseng, PgDel2 was dispersed in pericentromeric regions of all chromosomes. In tetraploid Panax, PgDel2 was concentrated at pericentromeric regions in 24 out of 48 chromosomes. Bar $=10 \mu \mathrm{m}$ 
$35 \%$ of the genome. Considering the size of PgDell being around $10 \mathrm{~kb}$, the increase in $P g D e l 1$ in P. quinquefolius accounts for almost $1 \mathrm{Gbp}$ of genome expansion. Compared to Panax species, A. elata had small amounts of PgDell, but was more abundant in PgTork (Lee et al. 2017). Major retrotransposons contributed to the speciation of the Panax species, which can be most evidently seen in the case of $P$. ginseng and $P$. quinquefolius. Although P. quinquefolius ( 4.9 Gb) diverged from $P$. ginseng $(\sim 3.6 \mathrm{~Gb})$ less than 1 mya, the genome size differs by $1.3 \mathrm{~Gb}$ which is most likely caused by the expansion of PgDell (Lee et al. 2017).

\section{Phylogenetic relationship among Panax genus and related species using sequence information}

$P$. ginseng and $P$. quinquefolius are allotetraploid species $(2 n=2 x=48)$ that grow in freezing winter regions while $P$. notoginseng, $P$. japonicus, $P$. vietnamensis, and $P$. stipuleanatus are diploid species inhabiting Asian countries in high altitude non-freezing regions. Meanwhile, P. trifolius is a diploid species that exist in the cold regions of North America. Complete chloroplast genome sequences and $45 \mathrm{~S}$ nrDNA of target species as well as Panax related species were obtained by using a de novo assembly method using low-coverage whole-genome sequence (dnaLCW) (Kim et al. 2015) and compared for genetic diversity and clues for evolution (Kim et al. 2017). Chloroplast genome and $45 \mathrm{~S}$ nrDNA sequences were compared to decipher the phylogeny of Panax species and related species and their classification. After diverging from the Apiaceae family 51 mya, Eleutherococcus species diverged from Panax and related genera around 10 mya. Then, Aralia elata, the closest relative to Panax species, diverged around 8 mya. Finally, the tetraploid $P$. ginseng and $P$. quinquefolius diverged around 1 mya after the second whole genome duplication event within the Panax genus at 2.2 mya (Fig. 2) (Kim et al. 2017). These results were generally in concordance with those of other reports (Lee et al. 2017; Kim et al. 2018). With the variation information derived from polymorphisms within the chloroplast genome sequence, molecular markers were designed and applied to distinguish the different species for authentication purposes which can be applied to various bioproducts (Nguyen et al. 2017, 2020).

\section{Migration and adaptation created the current genetic pool of Panax species}

Kim et al. (2018) suggests the current distribution of Panax species based on two intercontinental species migrations and cycles of ice ages and global warming. Ancestor diploid species of Panax could have started off inhabiting larger regions of Asia than those inhabited today. One diploid species, $P$. trifolius made the first intercontinental migration to the North American continent 6-7 mya. Then, glaciation caused the diploid species residing in Asia to face extinction because they do not have overwintering abilities. Two likely extinct ancestral diploid species created an allotetraploid ancestor of $P$. ginseng around 2 mya which may have survived in Northeast Asia while gaining overwintering properties. Meanwhile, most diploids could not survive in Northern Asia or lower altitudes in Southern Asia due to global warming, and the remaining moved up to higher altitudes in Southern Asia. While a tetraploid $P$. ginseng ancestor occupied the Northeast Asian regions, a second intercontinental migration occurred around 1 mya which made a possible ancestor of $P$. quinquefolius to North America during glacial migration (Fig. 4).

\section{Further studies}

Studies related to ginseng and related genera are ongoing. Although a $P$. ginseng reference genome is now available in literature (Jayakodi et al. 2018; Kim et al. 2018), this is still in a draft sequence level which needs considerable improvement to achieve chromosome-level assembly to be more reliable for sophisticated downstream analyses. Recent sequencing technologies such as Oxford Nanopore Sequencing Technologies and $\mathrm{Hi}-\mathrm{C}$, among others, have been recently adapted to improve the present genome assembly. These technologies have significantly refined the $P$. ginseng assembly to a pseudochromosome level, with super-scaffolds roughly representing the 24 chromosomes of $P$. ginseng that are being confirmed with oligo-FISH and genotyping by sequencing (GBS) methods (unpublished data).

Moreover, the evolution of the Araliaceae family is interesting to note because the Panax genus and the related genera are morphologically and ecologically diverse. Cytogenetic studies of 15 Araliaceae species revealed that genome sizes and chromosome numbers vary even within a monophyletic lineage, which calls for further research (unpublished data). Currently, chloroplast genome sequences of related species are available (Kim et al. 2016a, c; Chen et al. 2020), which can be utilized to further elucidate the relationship among these species and their evolution that leads to the different characteristics.

\section{Conclusion}

This review discussed the dynamic evolutionary history of Panax species that was revealed by whole genome sequence data of $P$. ginseng as well as comparative analysis with other related species. $P$. ginseng genome went through two rounds 


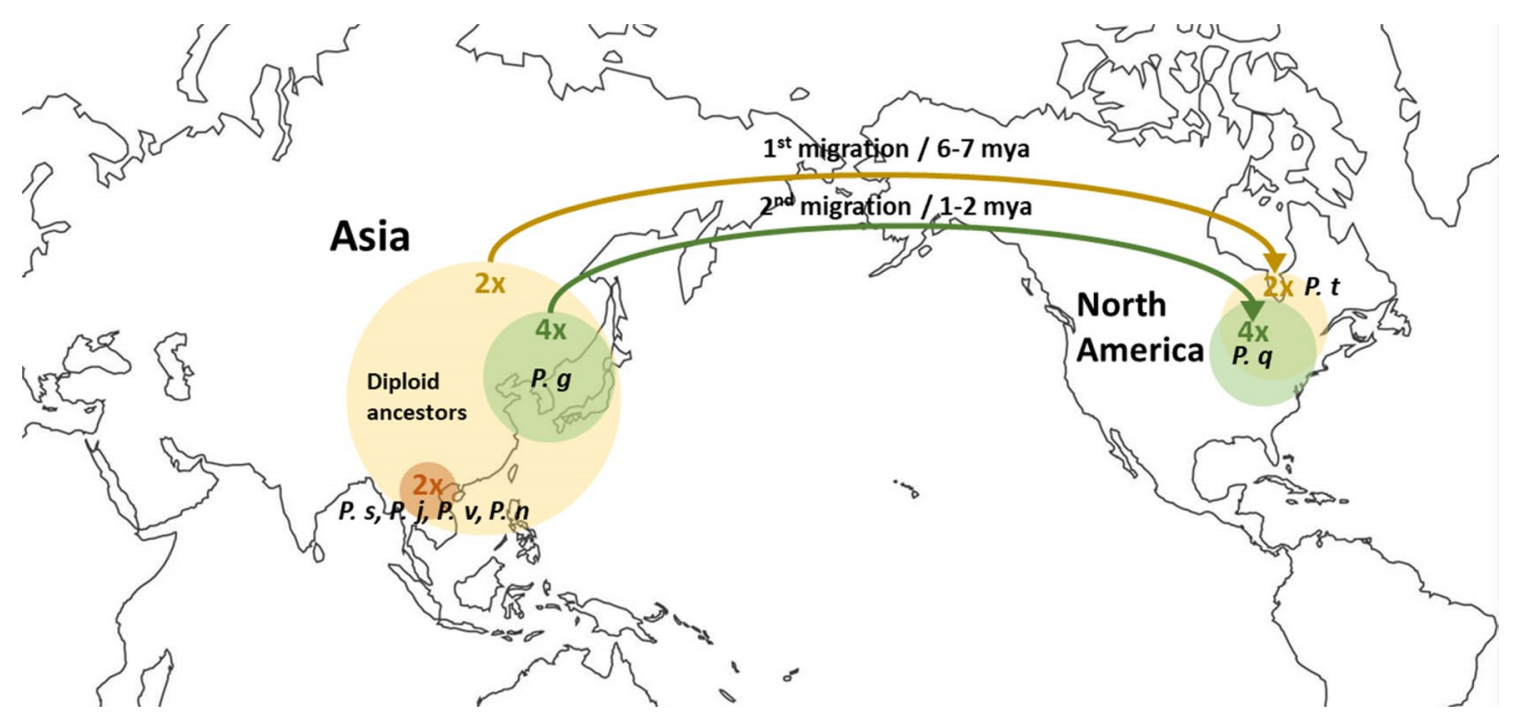

Fig. 4 Two intercontinental migration of Panax species recreated based on Kim et al. (2018). P. g P. ginseng, P. s P. stipuleanatus, P. j P. japonicas, P. v P. vietnamensis, P. n P. notoginseng, P. t P. trifolius, P. q P. quinquefolius

of whole genome duplication events throughout evolution that separately occurred after diverging from the closest family Apiaceae. Duplication events resulted in paralogous blocks within the genome that shows high sequence similarity. Sequence information such as repeat elements, paralogous genes, organellar genomes, and molecular cytogenetic tools were utilized to decipher the relationship among Panax species and detect clues for evolution and speciation. These results provide fundamental information for further ginseng research that can be used in various future studies such as molecular assisted breeding, and the understanding of evolutionary history of the Panax genus with other genera in the Araliaceae family.

Acknowledgements This research was supported by "Cooperative Research Program for Agriculture Science and Technology Development (Project No. PJ01311901)", Rural Development Administration, Republic of Korea.

Open Access This article is licensed under a Creative Commons Attribution 4.0 International License, which permits use, sharing, adaptation, distribution and reproduction in any medium or format, as long as you give appropriate credit to the original author(s) and the source, provide a link to the Creative Commons licence, and indicate if changes were made. The images or other third party material in this article are included in the article's Creative Commons licence, unless indicated otherwise in a credit line to the material. If material is not included in the article's Creative Commons licence and your intended use is not permitted by statutory regulation or exceeds the permitted use, you will need to obtain permission directly from the copyright holder. To view a copy of this licence, visit http://creativecommons.org/licenses/by/4.0/.

\section{References}

Akpinar BA, Biyiklioglu S, Alptekin B, Havránková M, Vrána J, Doležel J, Distelfeld A, Hernandez P, IWGSC, Budak H (2018) Chromosome-based survey sequencing reveals the genome organization of wild wheat progenitor Triticum dicoccoides. Plant Biotechnol J 16:2077-2087

Chen W, Kui L, Zhang G, Zhu S, Zhang J, Wang X, Yang M, Huang H, Liu Y, Wang Y, Li Y (2017) Whole-genome sequencing and analysis of the Chinese herbal plant Panax notoginseng. Mol Plant 10:899-902

Chen S, Xu Y, Liang D, Wang R (2020) The complete chloroplast genome of Eleutherococcus trifoliatus (Araliaceae): a wild edible plant in the coastal region of South China. Mitochondrial DNA B Resour 5:513-514

Choi KT (2008) Botanical characteristics, pharmacological effects and medicinal components of Korean Panax ginseng CA Meyer. Acta Pharmacol Sin 29:1109-1118

Choi YE, Yang DC, Park JC, Soh WY, Choi KT (1998) Regenerative ability of somatic single and multiple embryos from cotyledons of Korean ginseng on hormone-free medium. Plant Cell Rep 17:544-551

Choi HI, Kim NH, Lee J, Choi BS, Do Kim K, Park JY, Lee SC, Yang TJ (2013) Evolutionary relationship of Panax ginseng and $P$. quinquefolius inferred from sequencing and comparative analysis of expressed sequence tags. Genet Resour Crop Evol 60:1377-1387

Choi HI, Waminal NE, Park HM, Kim NH, Choi BS, Park M, Choi D, Lim YP, Kwon SJ, Park BS, Kim HH (2014) Major repeat components covering one-third of the ginseng (Panax ginseng CA Meyer) genome and evidence for allotetraploidy. Plant J 77:906-916

International Wheat Genome Sequencing Consortium (IWGSC) (2014) A chromosome-based draft sequence of the hexaploid bread wheat (Triticum aestivum) genome. Science 345:1251788

Doležel J, Vrána J, Cápal P, Kubaláková M, Burešová V, Šimková H (2014) Advances in plant chromosome genomics. Biotechnol Adv 32:122-136 
Fan G, Liu X, Sun S, Shi C, Du X, Han K, Yang B, Fu Y, Liu M, Seim I, Zhang H (2020) The chromosome level genome and Genome-Wide Association Study for the agronomic traits of Panax notoginseng. Iscience 23:101538

Grushwitsky IV (1961) Ginseng: the aspects of biology. Nauka, Leningrad (In Russian)

Jang W, Kim NH, Lee J, Waminal NE, Lee SC, Jayakodi M, Choi HI, Park JY, Lee JE, Yang TJ (2017) A glimpse of Panax ginseng genome structure revealed from ten BAC clone sequences obtained by SMRT sequencing platform. Plant Breed Biotechnol 5:25-35

Jayakodi M, Lee SC, Park HS, Jang W, Lee YS, Choi BS, Nah GJ, Kim DS, Natesan S, Sun C, Yang TJ (2014) Transcriptome profiling and comparative analysis of Panax ginseng adventitious roots. J Ginseng Res 38:278-288

Jayakodi M, Choi BS, Lee SC, Kim NH, Park JY, Jang W, Lakshmanan M, Mohan SV, Lee DY, Yang TJ (2018) Ginseng genome database: an open-access platform for genomics of Panax ginseng. BMC Plant Biol 18:62

Jiang Z, Tu L, Yang W, Zhang Y, Hu T, Ma B, Lu Y, Cui X, Gao J, Wu X, Tong Y, Zhou J, Song Y, Liu Y, Liu N, Huang L, Gao $\mathrm{W}$ (2020) The chromosome-level reference genome assembly for Panax notoginseng and insights into ginsenoside biosynthesis. Plant Commun 1:100113

Kim K, Lee SC, Lee J, Yu Y, Yang K, Choi BS, Koh HJ, Waminal NE, Choi HI, Kim NH et al (2015) Complete chloroplast and ribosomal sequences for 30 accessions elucidate evolution of Oryza AA genome species. Sci Rep 5:15655

Kim K, Lee J, Lee SC, Kim NH, Jang W, Kim S, Sung S, Lee J, Yang TJ (2016a) The complete chloroplast genome of Eleutherococcus gracilistylus (WW Sm.) SY Hu (Araliaceae). Mitochondrial DNA A 27:3741-3742

Kim K, Lee SC, Lee J, Kim NH, Jang W, Yang TJ (2016b) The complete chloroplast genome sequence of Panax quinquefolius (L.). Mitochondrial DNA A 27:3033-3034

Kim K, Lee SC, Yang TJ (2016c) The complete chloroplast genome sequence of Dendropanax morbifera (Leveille). Mitochondrial DNA A 27:2923-2924

Kim K, Dong J, Wang Y, Park JY, Lee SC, Yang TJ (2017) Evolution of the Araliaceae family inferred from complete chloroplast genomes and 45S nrDNAs of 10 Panax-related species. Sci Rep 7:1-9

Kim NH, Jayakodi M, Lee SC, Choi BS, Jang W, Lee J, Kim HH, Waminal NE, Lakshmanan M, Van Nguyen B et al (2018) Genome and evolution of the shade-requiring medicinal herb Panax ginseng. Plant Biotechnol J 16:1904-1917

Lee J, Waminal NE, Choi HI, Perumal S, Lee SC, Jang W, Kim NH, Gao LZ, Yang TJ (2017) Rapid amplification of four retrotransposon families promoted speciation and genome size expansion in the genus Panax. Sci Rep 7:1-9

Lysák M, Č́íUhalíková J, Kubaláková M, Šimková H, Künzel G, Doležel J (1999) Flow karyotyping and sorting of mitotic chromosomes of barley (Hordeum vulgare L.). Chromosome Res 7:431-444

Nguyen VB, Park HS, Lee SC, Lee J, Park JY, Yang TJ (2017) Authentication markers for five major Panax species developed via comparative analysis of complete chloroplast genome sequences. J Agric Food Chem 65:6298-6306

Nguyen VB, Giang VN, Waminal NE, Park HS, Kim NH, Jang W, Lee J, Yang TJ (2020) Comprehensive comparative analysis of chloroplast genomes from seven Panax species and development of an authentication system based on species-unique single nucleotide polymorphism markers. J Ginseng Res 44:135-144

Pinkel D, Straume T, Gray JW (1986) Cytogenetic analysis using quantitative, high-sensitivity, fluorescence hybridization. Proc Natl Acad Sci USA 83:2934-2938

Plunkett G, Soltis DE, Soltis PS (1996) Higher level relationships of Apiales (Apiaceae and Araliaceae) based on phylogenetic analysis of $r b c \mathrm{~L}$ sequences. Am J Bot 83:499-515

Schorger AW (1969) Ginseng: a pioneer resource. Wis Acad Sci Arts Lett 57:65-74

Van Duy N, Le Ngoc TR, Chinh ND, Van Tien TR (2016) A new variety of Panax (Araliaceae) from Lam Vien Plateau, Vietnam and its molecular evidence. Phytotaxa 277:47-58

Waminal NE, Park HM, Ryu KB, Kim JH, Yang TJ, Kim HH (2012) Karyotype analysis of Panax ginseng CA Meyer, 1843 (Araliaceae) based on rDNA loci and DAPI band distribution. Comp Cytogenet 6:425

Waminal NE, Choi HI, Kim NH, Jang W, Lee J, Park JY, Kim HH, Yang TJ (2017) A refined Panax ginseng karyotype based on an ultra-high copy 167-bp tandem repeat and ribosomal DNAs. J Ginseng Res 41:469-476

Wen J, Zimmer EA (1996) Phylogeny and biogeography of Panax L. (the ginseng genus, Araliaceae): inferences from ITS sequences of nuclear ribosomal DNA. Mol Phylogenet Evol 6:167-177

Wen J, Plunkett GM, Mitchell AD, Wagstaff SJ (2001) The evolution of Araliaceae: a phylogenetic analysis based on ITS sequences of nuclear ribosomal DNA. Syst Bot 26:144-167

Xu J, Chu Y, Liao B, Xiao S, Yin Q, Bai R, Su H, Dong L, Li X, Qian $\mathrm{J}$ et al (2017) Panax ginseng genome examination for ginsenoside biosynthesis. Gigascience 6:gix093

Zhang D, Li W, Xia EH, Zhang QJ, Liu Y, Zhang Y, Tong Y, Zhao Y, Niu YC, Xu JH, Gao LZ (2017) The medicinal herb Panax notoginseng genome provides insights into ginsenoside biosynthesis and genome evolution. Mol Plant 10:903-907

Zhang H, Abid S, Ahn JC, Mathiyalagan R, Kim YJ, Yang DC, Wang Y (2020) Characteristics of Panax ginseng cultivars in Korea and China. Molecules 25:2635

Zhuravlev YN, Koren OG, Reunova GD, Muzarok TI, Gorpenchenko TY, Kats IL, Khrolenko YA (2008) Panax ginseng natural populations: their past, current state and perspectives. Acta Pharmacol Sin 29:1127-1136

Publisher's Note Springer Nature remains neutral with regard to jurisdictional claims in published maps and institutional affiliations. 